\title{
Almacenamiento refrigerado de piña mínimamente procesada. Cambios en atributos físico-químicos y sensoriales
}

\author{
Saúl Dussán-Sarria ${ }^{(1) \star}$, María C. Rivera ${ }^{(1)}$, y Carlos A. García-Mogollon ${ }^{(2)}$ \\ (1) Facultad de Ingeniería y Administración, Departamento de Ingeniería, Universidad Nacional de Colombia. Sede \\ Palmira. A.A. 237. Palmira, Valle del Cauca-Colombia. (correo-e: sdussan@unal.edu.co; mcriverao@unal.edu.co) \\ (2) Universidad de Sucre. Sincelejo, Sucre, Colombia. (correo-e: carlos.garcia@unisucre.edu.co)
}

* Autor a quien debe ser dirigida la correspondencia.

Recibido Abr. 12, 2019; Aceptado Jun. 13, 2019; Versión final Ago. 12, 2019, Publicado Abr. 2020

\begin{abstract}
Resumen
El objetivo de este trabajo fue evaluar los cambios de calidad durante el almacenamiento refrigerado ocasionados por el tipo de corte y tipo de empaque en piña 'Oro Miel'. La piña es un fruto tropical apreciado por su sabor y color propicio para la aplicación de la tecnología de procesamiento mínimo. Los frutos fueron seleccionados, clasificados, higienizados, la cáscara, corona y corazón fueron retiradas, y se obtuvo cortes en cuartos de rodaja, julianos y cubos, empacados en cajas PET, bandejas de poliestireno con PVC y bolsas de polietileno para vacío. Durante 15 días de almacenamiento a $5 \pm 1^{\circ} \mathrm{C}$ y $85-90 \%$ de humedad relativa, se evaluó el porcentaje de jugo exudado, parámetros de color y análisis sensorial, aplicando estadística descriptiva y análisis de varianza. La piña en rodajas, sin ningún tipo de recubrimiento comestible, envasada al vacío y refrigerada es la condición que proporcionó los mejores atributos de calidad, potencializando una vida comercial hasta por 12 días.
\end{abstract}

Palabras clave: Ananas comosus (L.) Merr; cuarta gama; calidad de frutas; vida útil; atributos sensoriales

\section{Refrigerated storage of fresh-cut pineapple fruit. Changes in physical-chemical and sensory attributes}

\begin{abstract}
The objective of this work was to evaluate the changes generated during the refrigerated storage caused by the type of cutting and type of packing in 'Honey Gold' pineapple fruit Pineapple is a tropical fruit appreciated for its flavor and color suitable for application of fresh-cut technology. The fruits were selected, sorted, sanitized, shell, crown and heart were removed and cuts in slices, strips and pineapple cubes were obtained, packed in PET boxes, polystyrene trays with PVC and vacuum polyethylene bags. During 15 days of cold storage at $5 \pm 1{ }^{\circ} \mathrm{C}$ and $85-90 \%$ of relative humidity, the percentage of leakage juice, color parameters and sensory analysis were evaluated, applying descriptive statistics and variance analysis. The pineapple sliced, without any type of edible coating, vacuum packed and refrigerated is the condition that provided the best quality attributes, potentiating a shelf-life up to 12 days.
\end{abstract}

Keywords: Ananas comosus (L.) Merr; fresh-cut; fruit quality; shelf-life, sensory attributes 


\section{INTRODUCCIÓN}

La piña, (Ananas comosus) es una de las frutas tropicales de mayor producción en el mundo después del banano y los cítricos. La piña se produce en regiones tropicales y subtropicales con una producción mundial de 25,4 millones de toneladas y en Colombia de 950 mil toneladas en 2018 (Agronet, 2018), encontrándose entre las 15 frutas más consumidas en el país. Se observa una tendencia al crecimiento en la producción en el mundo siendo la variedad Oro Miel o Honey Gold la más apreciada a nivel mundial. El ministerio de Agricultura estima que el $30 \%$ de la producción de piña cumple con los parámetros técnicos de exportación y transportación y el otro se $70 \%$ se comercializa en el país. En 2018 estas exportaciones alcanzaron US\$2,5 millones. Respecto al consumo, el $49 \%$ se consume en fresco, el $49 \%$ se transforma en elaboración de concentrados azucarados y conservas: mientras, el $2 \%$ restante se exporta. La piña 'Oro Miel' posee forma cilíndrica, simétrica y uniforme, pericarpio entre color amarillo-naranja, pulpa de color amarillo, dulce, compacta y fibrosa con un peso promedio de 1,3 a 2,5 kg, presenta un elevado contenido de ácido ascórbico, azúcares y ácido ascórbico (Chan et al., 2003).

Se utilizan técnicas que se combinan sinérgicamente limitando el crecimiento microbiano y el metabolismo del producto, entre estas técnicas están el enfriamiento rápido, la refrigeración, la tecnología de atmosfera modificada y recubrimientos comestibles (Montero-Calderón et al., 2008; Sanjinez-Argandoña et al., 2010; Dussán-Sarria et al., 2014; Dussán-Sarria et al., 2015). Inhibidores de acción del etileno combinados con la refrigeración han sido aplicados también para evitar esos cambios indeseables en la piña y aumentar su vida útil (Rojas et al., 2007; Rangel y López, 2012), otros tratamientos como la aplicación de recubrimientos en combinación con antioxidantes y diferentes condiciones de empacado también han sido evaluados (MonteroCalderón et al., 2008). El procesamiento mínimo es una tecnología que combina dos importantes aspectos para el consumidor, uno es el concepto de "ready to eat" - listo para consumir- y dos, mantener las características de una fruta fresca (Koidis et al., 2012; Barbosa et al., 2013). Existe un mercado en crecimiento de las frutas mínimamente procesadas por ser consideradas por el consumidor un alimento listo para una dieta saludable, por ser un producto conveniente, por la reducción de costos originado por la eliminación de las partes no comestibles del fruto y la apreciación de su sabor y suculencia (Marrero y Kader, 2006; Escobar et al., 2014; Treviño-Garza et al., 2017). Sin embargo, las operaciones de higienizado, pelado y cortado resultan en una reducción de la vida útil respecto a la fruta madura fisiológicamente.

Frutas frescas cortadas en diferentes formas dependiendo de la estructura de la fruta, comercializadas en empaques plásticos y refrigeradas son alternativas para conservar las propiedades organolépticas y nutricionales inherentes típicas de la frutas (Turazzi et al., 2017). Durante y después del procesamiento mínimo de piña, el tipo de corte y el tipo de empaque para su almacenamiento tienen un efecto directo sobre su fisiología y conservación de la calidad del producto vegetal. La pulpa expuesta al medio ambiente presenta oscurecimiento enzimático que afecta su calidad sensorial, puede evidenciar crecimiento microbiano y acumulación de líquido exudado dentro del empaque (Soliva-Fortunuy y Martín-Belloso, 2003). La piña mínimamente procesada tiene una vida útil corta en torno de 5 a 7 días almacenada a temperatura entre 1 y $7^{\circ} \mathrm{C}$, vida útil reducida principalmente por procesos de degradación fisiológicos y microbianos (Pan et al., 2015). El objetivo de este estudio fue determinar el efecto del tipo de empaque en bandeja de poliestireno expandido, cajas de polietileno tereftalato y bolsas de polietileno de baja densidad; $y$ tipo de corte en cuartos de rodajas, cubos y julianas en el color, jugo exudado y aceptación sensorial de la piña mínimamente procesada, almacenada en ambiente refrigerado.

\section{MATERIALES Y MÉTODOS}

La metodología se presenta en varias subsecciones, para una mayor claridad: local y materia prima, procesamiento mínimo, empaque y almacenamiento, porcentaje de jugo exudado, medición del color, análisis sensorial y el respectivo análisis estadístico.

\section{Local y materia prima}

Los frutos de piña variedad Oro Miel fueron proporcionados por la empresa Oriente S.A. en el corregimiento Barrancas de Palmira, Valle del Cauca, Latitud: 3,517, Longitud: -76,3, $3^{\circ} 31^{\prime} 1^{\prime \prime}$ Norte, $76^{\circ} 18^{\prime} 0^{\prime \prime}$ Oeste, Temperatura $23^{\circ} \mathrm{C}, 1000$ m.s.n.m., $40 \%$ HR. Los frutos fueron trasladados al Laboratorio de Frutas y Hortalizas de la Universidad Nacional de Colombia Sede Palmira. Los frutos fueron seleccionados y clasificados en estado de madurez 3 , el cual fue definido preliminarmente como el más adecuado para el procesamiento mínimo, para esta clasificación se utilizó la escala de maduración referenciada en la norma técnica colombiana NTC 729-1 (ICONTEC, 1996). 


\section{Procesamiento mínimo}

Piñas enteras fueron lavadas y desinfectadas con solución de hipoclorito de sodio a $100 \mu$ l..$^{-1}$ durante 10 minutos (Sothornvit y Rodsamran, 2008). Los frutos enteros fueron almacenados a $10^{\circ} \mathrm{C}$ durante 12 horas previamente al procesamiento mínimo. De forma manual, siguiendo las recomendaciones de buenas prácticas de manufactura (BPM) en alimentos, se retiraron la corona, epicárpio y corazón, y fueron obtenidos 3 tipos corte de la pulpa: cuartos de rodajas (aproximadamente $37 \mathrm{~g}$ cada uno), cubos de $2 \mathrm{~cm} \times 2 \mathrm{~cm} \times 2 \mathrm{~cm}$ y julianas de $6 \mathrm{~cm} \times 2 \mathrm{~cm} \times 2 \mathrm{~cm}$. Los cortes de piña fueron higienizados con inmersión en solución de hipoclorito de sodio a $20 \mu \mathrm{ll} \mathrm{I}^{-1}$ durante 2 minutos. Los cortes más homogéneos fueron seleccionados y sumergidos en solución de cloruro de calcio $\left(\mathrm{CaCl}_{2}\right)$ al $1 \%(\mathrm{w} / \mathrm{w})$, ácido cítrico al $1 \%(\mathrm{w} / \mathrm{w})$, ácido ascórbico al $1 \%(\mathrm{w} / \mathrm{w})$. Con los ácidos orgánicos se esperaba impedir el oscurecimiento enzimático y conservar su firmeza (Robles et al, 2007). Seguidamente todos los cortes del fruto fueron colocados dentro de bolsas perforadas de naylon y sometidos a centrifugación de 2800 rpm durante 15 segundos en centrifuga Kohinoor A655 con el fin de eliminar su exceso de agua.

\section{Empaque y almacenamiento}

Muestras de $150 \mathrm{~g}$ fueron dispuestas en cada uno de los siguientes envases: bandeja de poliestireno expandido de $10 \mathrm{~cm} \times 10 \mathrm{~cm} \times 5 \mathrm{~cm}$ envuelta en policloruro de vinilo PVC de $14 \mu \mathrm{m}$, cajas de polietileno tereftalato PET de $11 \mathrm{~cm} \times 9 \mathrm{~cm} \times 2,5 \mathrm{~cm}$ y bolsas de polietileno de baja densidad PEBD de $15 \mathrm{~cm} \times 20 \mathrm{~cm}$ y $70 \mu \mathrm{m}$. Las respectivas permeabilidades son: $\mathrm{PVC}$ al $\mathrm{CO}_{2}$ de $5.183 \mathrm{~cm}^{3} \mathrm{~m}^{-2}$ día-1 y al $\mathrm{O}_{2}$ de $14.803 \mathrm{~cm}^{3} \mathrm{~m}^{-2}$ día1; PET al CO 2 de $112 \mathrm{~cm}^{3} \mathrm{~m}^{-2}$ día-1 y al $\mathrm{O}_{2}$ de $3.940 \mathrm{~cm}^{3} \mathrm{~m}^{-2}$ día-1 y PEBD al $\mathrm{CO}_{2}$ de $107 \mathrm{~cm}^{3} \mathrm{~m}^{-2}$ día-1 y al $\mathrm{O}_{2}$ de $39 \mathrm{~cm}^{3} \mathrm{~m}^{-2} \mathrm{día}^{-1}$. Los cortes de fruto empacados fueron almacenados a $5 \pm 1{ }^{\circ} \mathrm{C}$ y $85-90 \%$ de $\mathrm{HR}$ (Silva et al., 2005). Cada 3 días y durante 15 días de almacenamiento refrigerado fueron evaluados los siguientes atributos de calidad:

\section{Porcentaje de jugo exudado}

El efecto del corte y empaque sobre la pérdida de jugo fue medido en relación al $100 \%$ del peso de la muestra de fruto en cada envase de acuerdo al método propuesto por Marrero y Kader (2006) en el cual el envase se inclina $20^{\circ}$ durante 5 minutos para medir la cantidad de jugo exudado. En este estudio los envases rígidos, es decir el PET y la bandeja de poliestireno, se mantuvieron a esa misma inclinación durante todo el periodo de experimentación.

\% jugo exudado $=\frac{P_{L}}{P_{i}} \times 100$

\section{Donde:}

PL corresponde al peso del jugo exudado en el periodo de análisis

$\mathrm{P}$ icorresponde al peso inicial del contendió de fruta en el envase

\section{Medición del color}

El color de la superficie de los trozos de piña se midió utilizando un colorímetro Marca Konica Minolta, Modelo: CR - 400, escala $\left(L^{*}, a^{*}, b^{*}\right)$, D65 como fuente luminosa y $2^{\circ}$ ángulo de observación, Las mediciones de color se expresaron en términos de luminosidad $L^{*}\left(L^{*}=0\right.$ para el negro y $L^{*}=100$ para el blanco), coordenada $a^{*}$ (verde [-], rojo [+]) y coordenada $b^{*}$ (azul[-], amarillo [+]). A partir de estas coordenadas, fueron calculadas los atributos de color croma $\left(\mathrm{C}^{*}\right)$ y tono $\left(\mathrm{H}^{*}\right)$ utilizando la ecuación 2 y la ecuación 3 , respectivamente (Chiumarelli et al., 2011). El porcentaje de variación de tono se determinó a través de la ecuación 4.

$$
\begin{aligned}
& C=\left(a^{2}+b^{2}\right)^{1 / 2} \\
& H=\arctan \left(\frac{b}{a}\right) \\
& \%(\Delta H)=\left(\frac{H_{\text {final }}-H_{\text {inicial }}}{H_{\text {inicial }}}\right) \times 100
\end{aligned}
$$

En donde $\mathrm{H}_{\text {inicial }}$ es el tono al inicio y $\mathrm{H}_{\text {final }}$ es el tono en cada periodo de almacenamiento. 


\section{Análisis sensorial}

El análisis sensorial afectivo fue realizado en los días 0,10 y 12 de almacenamiento con un grupo de 30 panelistas no entrenados. Los atributos sensoriales de los cortes de piña aroma, sabor y aceptación general fueron evaluados. Fue utilizada la escala hedónica afectiva de 5 puntos que define el grado de satisfacción donde: $1=$ no me gusta, 2 = me disgusta ligeramente, $3=$ ni me gusta ni me disgusta, $4=$ me gusta ligeramente, 5 = me gusta mucho. Los cortes se consideraron sensorialmente aceptables con notas iguales o superiores a 3.

Análisis estadístico

Se utiliza un diseño delineamiento experimental completamente al azar con arreglo factorial $3 \times 3 \times 6$ correspondiente a los factores tipos de corte con tres niveles, tipos de empaque con tres niveles y tiempos de almacenamiento con seis niveles $(0,3,6,9,12,15$ días). A los datos se les aplicó análisis de varianza (ANOVA) y comparación de medias utilizando la prueba de Tukey $(p<0,05)$. Fue utilizado el programa estadístico IBM SPSS v. 20.0. La designación de los 9 tratamientos y sus respectivas descripciones son descritas en la Tabla 1.

Tabla 1: Denominación y descripción de los tratamientos evaluados.

\begin{tabular}{|l|l|}
\hline Denominación & Descripción \\
\hline CR-PET & Piña cortada en cuartos de rodaja envasada en caja PET \\
\hline C-PET & Piña cortada en cubos envasada en caja PET \\
\hline J-PET & Piña cortada en julianas envasada en caja PET \\
\hline CR-PS & Piña cortada en cuartos de rodaja envasada en bandeja de poliestireno recubierta con plástico PVC \\
\hline C-PS & Piña cortada en cubos envasada en bandeja de poliestireno recubierta con plástico PVC \\
\hline J-PS & Piña cortada en julianas envasada en bandejas de poliestireno recubierta con plástico PVC \\
\hline CR-V & Piña cortada en cuartos de rodaja envasada en bolsa de polietileno al vacío \\
\hline C-V & Piña cortada en cubos envasada en bolsa de polietileno al vacío \\
\hline J-V & Piña cortada en julianas envasada en bolsa de polietileno al vacío. \\
\hline
\end{tabular}

\section{RESULTADOS Y DISCUSIÓN}

Tanto en los cambios de valores del porcentaje de jugo exudado como en los valores de los atributos de color existe efecto del tipo de empaque y no del tipo de corte $(p<0,05)$. En los empaques PET y bandeja de polietileno con PVC no se presentó líquido exudado, mientras que en la bolsa a vacío si hubo (Tabla 2). En el día 15 de almacenamiento la mayor acumulación de líquido exudado se presentó en el tratamiento CR-V con un valor del $16 \pm 3,61 \%$, seguido de J-Bolsa vacío con $13,93 \pm 2,39 \%$ y la menor acumulación en C-V con $11,5 \pm 4,64 \%$. En el día 12 de almacenamiento (periodo límite de conservación de acuerdo al análisis sensorial posterior), los tratamientos $\mathrm{CR}-\mathrm{V}$ y J-V presentaron los mismos valores de líquido exudado $(p<0,05)$ mientras que el tratamiento C-Bolsa vació exhibió la menor acumulación. A pesar que la piña en cuartos de rodaja al vacío presentó presencia de jugo exudado, seguramente debido que la diferencia de presión de vapor generó una presión osmótica en contra, el fruto mantuvo el color y aroma característico. Además de la exudación normal de la piña, por sus características comunes, esto también se puede presentar por los daños mecánicos que se le han ocasionado a la pulpa, pues particularmente en este tipo de matriz biológica cuando se hace un corte hay rompimiento de células se acelera su deterioro y provoca la fuga de fluidos.

Los valores de luminosidad y croma (Tabla 3 ) en general disminuyeron durante el tiempo de almacenamiento con excepción de los cortes de fruto empacados al vacío los cuales presentaron una tendencia al aumento a medida que se prolonga el almacenamiento como consecuencia de la exclusión de oxigeno contribuyendo a la estabilidad química de los carotenoides. La disminución de los valores de croma son el reflejo de la disminución de los valores de la coordenada $b^{*}$ que indican una tendencia a una tonalidad más oscuras. Al inicio del almacenamiento los cortes de piña empacados en PET y en poliestireno con PVC el valor medio general de luminosidad fue de $67,77 \pm 4,4$ y al día 12 de $52,88 \pm 1,2$ lo que representa una disminución del $22 \%$ en la luminosidad del fruto. Montero et al. (2008) observaron también una reducción del $22 \%$ en los valores del parámetro L* en piña mínimamente procesada empacada en bandeja de polipropileno. González et al. (2004) observaron una disminución significativa de los parámetros de color $L^{*}$ y $b^{*}$ durante 14 días de almacenamiento de rodajas de piña tratadas con ácido ascórbico $(0,05 \%)$ y empacadas en bandeja de poliestireno. De acuerdo con Antoniolli et al. (2003) el cloruro de calcio aplicado en trozos de piña provoca también un descenso del parámetro $L^{*}$ indicando cierto oscurecimiento de las muestras al igual que el pardeamiento por la acción de la enzima polifenoloxidasa, pérdida gradual de agua o deshidratación superficial (Pérez et al., 2016; Djioua et al., 2010). 
Tabla 2: Efecto del tipo de corte en los valores de jugo exudado durante el almacenamiento de piña mínimamente procesada empacada en bolsa de vacío

\begin{tabular}{|c|c|c|}
\hline Tipo corte & Días & Jugo exudado (\%) \\
\hline \multirow{4}{*}{ Cuartos de rodaja } & 0 & $0 \pm 0$ \\
\cline { 2 - 3 } & 6 & $9,67 \pm 3,23$ \\
\cline { 2 - 3 } & 9 & $9,67 \pm 3,23$ \\
\cline { 2 - 3 } & 12 & $12,53 \pm 5,08$ \\
\cline { 2 - 3 } & 15 & $16 \pm 3,61$ \\
\hline \multirow{5}{*}{ Cubos } & 0 & $0 \pm 0$ \\
\cline { 2 - 3 } & 6 & $5,33 \pm 0,30$ \\
\cline { 2 - 3 } & 9 & $6,73 \pm 1,10$ \\
\cline { 2 - 3 } & 12 & $7,53 \pm 0,61$ \\
\cline { 2 - 3 } & 15 & $11,5 \pm 4,64$ \\
\hline \multirow{5}{*}{ Julianas } & 0 & $0 \pm 0$ \\
\cline { 2 - 3 } & 6 & $10,12 \pm 5,93$ \\
\cline { 2 - 3 } & 9 & $10,12 \pm 5,93$ \\
\cline { 2 - 3 } & 12 & $12,60 \pm 4,89$ \\
\cline { 2 - 3 } & 15 & $13,93 \pm 2,39$ \\
\hline
\end{tabular}

Tabla 3: Efecto del tipo de corte y empaque en los valores de luminosidad $\left(L^{*}\right)$ y croma $\left(C^{*}\right)$ durante el almacenamiento de piña mínimamente procesada

\begin{tabular}{|l|c|c|c|c|c|c|c|}
\hline \multirow{3}{*}{ Corte } & \multirow{2}{*}{ Dias } & \multicolumn{2}{|c|}{ Polietileno tereftalato } & \multicolumn{2}{c|}{ Poliestireno } & \multicolumn{2}{c|}{ Bolsa vacío } \\
\cline { 2 - 7 } & & $L^{*}$ & $C^{*}$ & $L^{*}$ & $C^{*}$ & $L^{*}$ & $C^{*}$ \\
\hline \multirow{5}{*}{$\begin{array}{l}\text { Cuartos } \\
\text { de rodaja }\end{array}$} & 0 & $67,77 \pm 4,4$ & $49,36 \pm 8,57$ & $67,77 \pm 4,4$ & $49,37 \pm 8,57$ & $67,77 \pm 4,4$ & $49,36 \pm 8,57$ \\
\cline { 2 - 8 } & 6 & $60,03 \pm 6,87$ & $35,13 \pm 0,7$ & $53,98 \pm 1,46$ & $32,37 \pm 2,32$ & $46,05 \pm 1,08$ & $39,58 \pm 5,34$ \\
\cline { 2 - 8 } & 12 & $54,29 \pm 4,40$ & $33,82 \pm 4,27$ & $51,64 \pm 3,07$ & $26,75 \pm 2,43$ & $44,51 \pm 2,78$ & $35,81 \pm 8,01$ \\
\cline { 2 - 8 } & 15 & $49,31 \pm 0,58$ & $24,67 \pm 1,83$ & $56,45 \pm 4,20$ & $22,83 \pm 1,39$ & $49,68 \pm 0,32$ & $47,17 \pm 5,02$ \\
\hline \multirow{5}{*}{ Cubos } & 0 & $67,77 \pm 4,4$ & $49,36 \pm 8,57$ & $67,77 \pm 4,4$ & $49,36 \pm 8,57$ & $67,77 \pm 4,4$ & $49,36 \pm 8,57$ \\
\cline { 2 - 8 } & 6 & $56,90 \pm 5,75$ & $31,85 \pm 5,89$ & $54,42 \pm 7,66$ & $32,04 \pm 3,90$ & $44,78 \pm 3,77$ & $39,84 \pm 13,85$ \\
\cline { 2 - 8 } & 9 & $56,63 \pm 4,24$ & $38,89 \pm 10,34$ & $55,45 \pm 9,63$ & $32,91 \pm 5,43$ & $47,77 \pm 2,64$ & $39,17 \pm 7,53$ \\
\cline { 2 - 8 } & 12 & $55,81 \pm 0,95$ & $33,45 \pm 2,47$ & $60,87 \pm 3,52$ & $33,66 \pm 0,89$ & $46,26 \pm 0,84$ & $46,17 \pm 2,61$ \\
\cline { 2 - 8 } & 15 & $56,59 \pm 8,10$ & $30,05 \pm 8,69$ & $51,52 \pm 5,97$ & $20,55 \pm 2,40$ & $54,43 \pm 1,06$ & $48,51 \pm 2,66$ \\
\hline \multirow{5}{*}{ Julianas } & 0 & $67,77 \pm 4,4$ & $49,37 \pm 8,57$ & $67,77 \pm 4,4$ & $49,36 \pm 8,57$ & $67,77 \pm 4,4$ & $49,36 \pm 8,57$ \\
\cline { 2 - 8 } & 6 & $68,34 \pm 1,38$ & $38,72 \pm 3,40$ & $64,69 \pm 2,56$ & $36,35 \pm 0,96$ & $43,32 \pm 3,96$ & $32,77 \pm 7,86$ \\
\cline { 2 - 8 } & 9 & $55,23 \pm 1,43$ & $29,3 \pm 1,11$ & $52,80 \pm 4,04$ & $29,19 \pm 3,42$ & $45,49 \pm 2,15$ & $38,9 \pm 2,60$ \\
\cline { 2 - 8 } & 12 & $55,54 \pm 5,77$ & $83,65 \pm 85,3$ & $59,81 \pm 9,71$ & $30,25 \pm 4,13$ & $46,86 \pm 0,73$ & $49,11 \pm 0,90$ \\
\cline { 2 - 8 } & 15 & $54,96 \pm 5,30$ & $30,82 \pm 1,28$ & $47,33 \pm 7,28$ & $23,2 \pm 1,84$ & $51,60 \pm 1,88$ & $56,85 \pm 2,86$ \\
\hline
\end{tabular}

La disminución de los valores porcentaje de variación de tono (Tabla 4) respecto al tono inicial $(96,1 \pm 0,81)$ son el reflejo de la disminución de los valores de la coordenada $b^{*}$ que indican una tendencia a una tonalidad más oscuras, principalmente en el empaque de poliestireno dada su alta permeabilidad a los gases. Considerando el tipo de corte, los cuartos de rodaja empacadas al vacío mantuvieron la tonalidad inherente a la piña fresca y los cortes en juliana presentaron las mayores variaciones independientes del tipo de empaque.

La disminución progresiva de los valores de croma y tono indican el cambio de tonalidad amarilla a una tonalidad más oscura en la piña mínimamente procesada. Cambios en la coloración de la piña mínimamente procesada debida al efecto del empaque también fueron identificados por Rocculi et al. (2009). El deterioro de la apariencia respecto al color en vegetales mínimamente procesados según Chauhan et al. (2006) es muy susceptible debido a la pérdida acelerada de pigmentos, como los carotenos y flavonoides, encargados de dar el color amarillo a las frutas; lo cual, se evidencio por oscurecimiento, pérdida de intensidad y brillo en la 
piña, ocasionando pérdida de calidad de la fruta en los últimos días de almacenamiento. Los cortes de piña envasados en la bandeja de poliestireno envuelta con PVC y las cajas de PET presentaron un deterioro significativo en cuanto al color y apariencia física mientras el fruto empacado al vació proporcionó la mejor estabilidad de color y apariencia en general.

Tabla 4: Efecto del tipo de corte y empaque en la variación del tono $\Delta \mathrm{H}(\%)$ durante el almacenamiento de piña mínimamente procesada a los 15 días.

\begin{tabular}{|l|c|c|c|}
\hline \multicolumn{1}{|c|}{ Tipo de corte } & Polietileno tereftalato & Poliestireno & Bolsa vacio \\
\hline \multirow{2}{*}{ Cuartos de rodaja } & & & \\
\cline { 2 - 4 } & $0,39 \%^{\mathrm{a}}$ & $-3,86 \%^{\mathrm{d}}$ & $-0,28 \%^{\mathrm{g}}$ \\
\hline \multirow{2}{*}{ Cubos } & $-0,35 \%^{\mathrm{b}}$ & $-1,63 \%^{\mathrm{e}}$ & $2,41 \%^{\mathrm{h}}$ \\
\hline \multirow{2}{*}{ Julianas } & & & \\
\cline { 2 - 4 } & $0,87 \%^{\mathrm{c}}$ & $-5,80 \%^{\mathrm{f}}$ & $-3,10 \%^{\mathrm{i}}$ \\
\hline
\end{tabular}

\section{Evaluación Sensorial}

Durante el almacenamiento refrigerado, considerando todos los tratamientos, la piña MP exhibió una tendencia a la disminución en las notas sensoriales, es decir, a presentar menor aceptación. Estadísticamente se encontró un efecto significativo del tipo de empaque y el tipo de corte sobre los atributos sensoriales de aroma, sabor y aceptación general de la piña cortada $(p<0,05)$, es decir, que la calidad sensorial de la piña MP depende del tipo de empaque y del tipo de corte. En general las mayores notas sensoriales y mantenimiento de esos valores en el tiempo de almacenamiento fueron atribuidas a la piña en cuartos de rodaja y envasada al vacío $(\mathrm{CR}-\mathrm{V})$. La evaluación sensorial fue considerada hasta el día 12 de almacenamiento, periodo limite en el cual se obtuvieron notas sensoriales iguales o superiores a 3 (Figura 1).

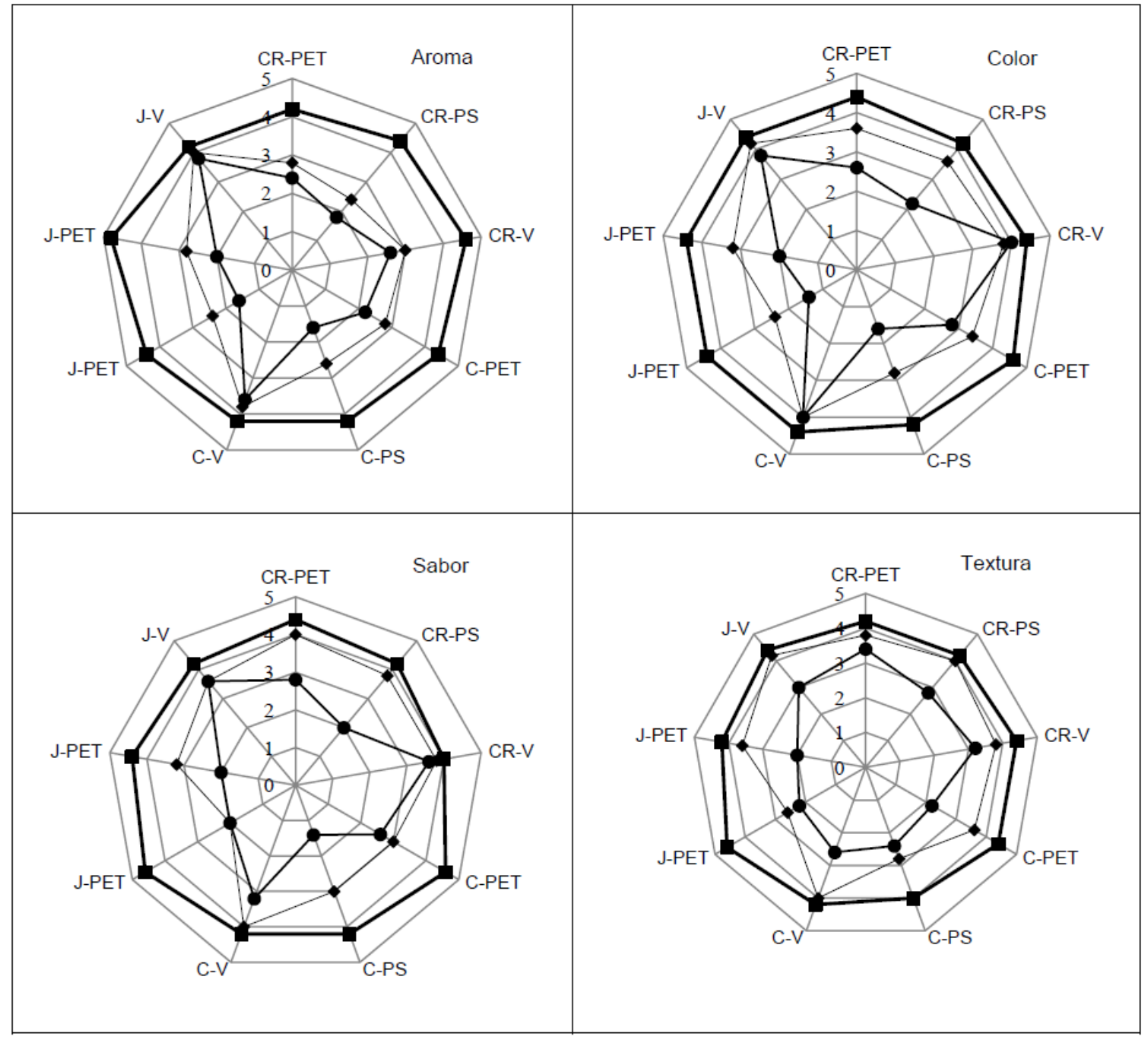

Fig. 1: Notas sensoriales de piña mínimamente procesada con diferentes tipos de corte, empaque y almacenada bajo refrigeración (días 0: $\mathbf{\bullet}, 10: \diamond$ y 12: •). 
La piña es un fruto tropical no climatérico, es decir que cuando almacenado, los cambios metabólicos derivados de la maduración o no suceden o son lentos. Estos cambios inevitables en la maduración fueron regulados por los tratamientos previos de antioxidantes, aplicación de calcio, empaque, refrigeración y las prácticas de inocuidad, de no emplearse estas tecnologías agroindustriales, en la piña mínimamente procesada, se presentará una rápida perecibilidad (García et al., 2011).

En este estudio se observó una decoloración del fruto originado en el proceso de maduración. Esta decoloración ya discutida en los cambios de los atributos de color y que tuvo incidencia en la evaluación sensorial fue observada en todos los cortes empacados en PET y poliestireno, ya los cortes empacados en bolsa de vacío mantuvieron el color amarillo característico. De la misma forma en los empaques PET y poliestireno, las notas sensoriales del aroma y sabor disminuyeron la aceptación debido a la presencia de sustancias aromáticas presentes en la pulpa, formando una mezcla de componentes orgánicos relacionados con el proceso de maduración avanzada (García et al., 2011). La piña, cuando almacenada mínimamente procesada, pierde el aroma característico de fruta fresca debido a la pérdida de compuestos aromáticosalifáticos, aldehídos, cetonas y compuestos menos polares, y contrastan con la aparición de compuestos azufrados, polares, alcoholes, cetonas y terpenos que generan aroma desagradable a medida que avanza el almacenamiento (Torri et al, 2010).

Spanier et al. (1998) mencionan que el sabor de la piña mínimamente procesada se puede perder durante el almacenamiento refrigerado debido a las reacciones enzimáticas que se suceden en el fruto y pueden generar sabor a fermentando. De acuerdo con Escobar (2014), la pérdida de textura en frutas mínimamente procesadas es asociada al deterioro fisiológico de pérdida de peso que probablemente se origina de la plasmólisis de las células vegetales, evidenciado a su vez por la pérdida de color. La piña mínimamente procesada variedad 'Pérola' puede preservar sus características de fruta fresca hasta el día 6 de almacenamiento, siendo factible desarrollar después de ese tiempo, un olor desagradable y sabor a fruta fermentada (Antoniolli et al., 2003). En el día 12 de almacenamiento, las mayores notas sensoriales de aceptación en cuanto a aroma, color, sabor y textura fueron otorgadas a la piña 'Oro Miel' en cuartos de rodajas envasada en bolsa de vacío (CR-V), resultado similar encontraron Dussán-Sarria et al. (2014) en piña "Manzana" mínimamente procesada.

\section{CONCLUSIONES}

Existe efecto del tipo de empaque en el porcentaje de jugo exudado y atributos de color y un efecto del tipo de empaque y tipo de corte en los atributos sensoriales durante el almacenamiento de piña 'Oro Miel' mínimamente procesada. La piña mínimamente procesada, cortada en rodajas y envasada al vacío en bolsas de polietileno es la condición más adecuada que proporciona los mejores atributos de calidad durante el almacenamiento y prolonga la vida útil hasta por 12 días.

\section{REFERENCIAS}

Agronet., Ministerio de Agricultura y Desarrollo Rural. República de Colombia (2018).

Antoniolli, L., Benedetti, B.C. y Souza Filho, M. S. M., Efeito do cloreto de cálcio na qualidade de abacaxi "Pérola" minimamente procesado, doi: 10.1590/S0100-204X2003000900012, Pesq. agropec. bras., 38(9), 1105-1110 (2003).

Barbosa, A. A. T., Araújo, H. G. S. y otros tres autores, Effects of Nisin-Incorporated Films on the Microbiological and Physicochemical Quality of Minimally Processed Mangoes, doi: https://doi.org/10.1016/j.ijfoodmicro.2013.04.004, International Journal of Food Microbiology, 164(2-3), 135-140 (2013).

Chan, Y.K., Coppens d'Eeckenbrugge, G. y Sanewski, G.M., Breeding and variety improvement, In The Pineapple: Botany, Production and Uses by D.P. Bartholomew, R.E. Paull y K.G. Rohrbach, pp 33-56 CABI Pub, New York, USA (2003).

Chauhan, O., Raju, P. y otros 3 autores, Modified/controlled Atmosphere Storage of Minimally Processed Mango Slices (var. Arka anmol), doi: 10.3923/ajft.2006.105.116, Rev. American Journal of Food Technology, 1(2), 105-116 (2006).

Chiumarelli, M., Ferrari, C. Sarantópoulos, C. y Hubinger, M., Fresh Cut 'Tommy Atkins' Mango Pre-Treated with Citric Acid and Coated with Cassava (Manihot esculenta crantz) Starch or Sodium Alginate, doi: 10.1016/j.ifset.2011.02.006, Innovative Food Science \& Emerging Technologies, 12(3), 381-387 (2011).

Djioua, T., Charles, F. y otros seis autores, Improving the Storage of Minimally Processed Mangoes (Mangifera indica L.) by Hot Water Treatments, doi: 10.1016/j.postharvbio.2008.10.006, Postharvest Biology and Technology, 52(2), 221-226 (2010).

Dussán-Sarria, S., Reyes-Cavache, P.M. y Hleap-Zapata, J.I., Efecto de un Recubrimiento Comestible y diferentes Tipos de Empaque en los Atributos Físico-químicos y Sensoriales de Piña 'manzana' Mínimamente Procesada, doi: 10.4067/S0718-07642014000500007, Inf. Tecnol., 25(5), 41-46 (2014).

Dussán-Sarria, S., Garcia-Mogollon, C. y Gutiérrez-Guzmán, N., Cambios Físico-Químicos y Sensoriales Producidos por el Tipo de Corte y Empaque en Zanahoria (Daucus carota L.) Mínimamente Procesada, doi: https://dx.doi.org/10.4067/S0718-07642015000300010, Inf. Tecnol., 26(3), 63-70 (2015). 
Escobar, A., Márquez, C., Restrepo, C. y Pérez, L., Aplicación de Tecnología de Barreras para la Conservación de Mezclas de Vegetales Mínimamente Procesados. doi: 10.15446/rfnam.v67n1.42652, Rev. Fac. Nac. Agron. Medellín, 67(1), 72387245 (2014).

García, Y., García, A., Hernández, A. y Pérez, J., Estudio de la Variación del Índice de Color durante la Conservación de la Piña Variedad Cayena Lisa a Temperatura Ambiente, ISSN 2071-0054, Rev Cie Téc Agr., 20(4), 12-16 (2011).

ICONTEC., Norma Técnica Colombiana. NTC 729-1. Frutas Frescas. piña. especificaciones, 1-10, Bogotá, Colombia (1996).

González-Aguilar, G.A., Ruiz-Cruz, S. y otros tres autores, Physiological and Quality Changes of Fresh-Cut Pineapple Treated with Antibrowning Agents, doi: 10.1016/j.Iwt.2003.10.007, Lebensm Wiss Technol., 37(3), 369-76 (2004).

Koidis, A., Rawson, A., Tuohy, M. y Brunton, N., Influence of Unit Operations on the Levels of Polyacetylenes in Minimally Processed Carrots and Parsnips: an Industrial Trial, doi: http://dx.doi.org/10.1016/j.foodchem.2011.11.128. Food Chemistry, 132(3), 1406-1412 (2012).

Marrero, A. y Kader, A.A., Optimal Temperature and Modified Atmosphere for Keeping Quality of Fresh-Cut Pineapples, doi: 10.1016/j.postharvbio.2005.10.017, Postharvest Biol. Technol., 39(2), 163-168 (2006).

Montero-Calderón, M., Rojas-Graü, M.A. y Martín-Belloso, O., Effect of Packaging on Quality and Shelf-Life of Fresh CutPineapple (Ananas Comosus), doi: 10.1016/j.postharvbio.2008.03.014, Postharvest Biology and Technology, 50(2-3), 182-189 (2008).

Pan, Y., Zhu, J. y Li, S., Effects of Pure Oxygen and Reduced Oxygen Modified Atmosphere Packaging on the Quality and Microbial Characteristics of Freshcut Pineapple, doi: 10.1051/ fruits/2015003, Fruits, 70(2), $101-108$ (2015).

Pérez, A.F., Aristizábal, I.D. y Restrepo, J.I., Conservación de Mango Tommy Atkins Mínimamente Procesado mediante la Aplicación de un Recubrimiento de Aloe Vera (Aloe Barbandensis Miller), doi: 10.17533/udea.vitae.v23n1a02, Vitae, 23(1), 65-77 (2016).

Rangel, M. y López, A., Cambios en Frutas Tropicales Frescas, Cortadas y Empacadas en Atmósfera Modificada durante su Almacenamiento en Refrigeración, Rev. Temas Selectos de Ingeniería de Alimentos, 6(2), 94-109 (2012).

Robles, M., Gorinstein, S. y otros cuatro autores, Frutos Tropicales Mínimamente Procesados: Potencial Antioxidante y su Impacto en la Salud, ISSN: 0378-1844, Interciencia, 32, 227 - 232 (2007).

Rocculi, R., Cocci, E. y otros tres autores, Effect of 1-MCP Treatment and $\mathrm{N}_{2} \mathrm{O}$ MAP on Physiological and Quality Changes of Fresh-Cut Pineapple, doi: 10.1016/j.postharvbio.2008.07.010, Postharvest Biol. Technol. 51, 371 -377 (2009).

Rojas-Graü, M.A., Tapia, M.S. y Martín-Belloso, O., Using Polysaccharide-Based Edible Coatings to Maintain Quality of Fresh-Cut Fuji Apples, doi: 10.1016/j.Iwt.2007.01.009, Food Sci. Technol., 41(1), 139-147 (2008).

Sanjinez-Argandoña, E.J., Branco, I.G., Takito, S. y Corbari, J., Influencia de la Deshidratación Osmótica y de la Adición de Cloruro de Calcio en la Conservación de Kiwis Minimamente Procesados. doi: 10.1590/S0101-20612010000500031, Food Sci. Technol., 30(suppl. 1), 205-209 (2010).

Silva, G. C., Maia, G.A. y otros cuatro autores, Efeito do Tipo de Corte nas Características Físico-Químicas do Abacaxi Pérola Minimamente Processado, doi: 10.1590/S0101-20612005000200006, Ciênc. Tecnol. Aliment., 25(2), 223-228 (2005).

Soliva-Fortuny, R.C. y Martín-Belloso, O., New Advances in Extending Shelf-Life of Fresh-Cut Fruits: a Review, doi: 10.1016/S0924-2244(03)00054-2, Trends Food Sci. Technol., 14(9), 341-353 (2003).

Sothornvit, R. y Rodsamran, P., Effect of a Mango Film on Quality of Whole and Minimally Processed Mangoes, doi: 10.1016/j.postharvbio.2007.08.005, Postharvest Biology and Technology, 47(3), 407-415 (2008).

Spanier, A., Flores, M. y otros cuatro autores, Fresh-Cut Pineapple (Ananas sp.) Flavor. Effect of Storage. doi: 10.1016/S0167-4501(98)80057-5, Dev. Food Sci. 40, 331-343. (1998).

Torri, L., Sinelli, N. y Limbo, S., Shelf Life Evaluation of Fresh-Cut Pineapple by Using an Electronic Nose. doi: 10.1016/j. postharvbio.2010.01.012, Postharvest Biol. Technol, 56(3), 239-245 (2010).

Treviño-Garza, M. Z., García, S. y otros tres autores, Layer-by-Layer Edible Coatings Based on Mucilages, Pullulan and Chitosan and Its Effect on Quality and Preservation On Fresh-Cut Pineapple (Ananas comosus), doi: 10.1016/j.postharvbio.2017.01.007, Postharvest Biology and Technology, 128, 63-75 (2017).

Turazzi, F.C., Merib, J. y otros cuatro autores, Evaluation of Volatile Profiles Obtained for Minimally-Processed Pineapple Fruit Samples During Storage by Headspace-Solid Phase Nicroextraction Gas Chromatography-Mass Spectrometry. doi: 10.1590/1678-457x.29316, Food Science and Technology, 37(4), 663-672 (2017). 